\title{
Effects of FHL1 and P21 on hypoxia-induced pulmonary vascular remodeling in neonatal rats
}

\author{
YANNA DU, JIANHUA FU, LI YAO, DAN ZHANG, NA LIU and XINDONG XUE \\ Department of Pediatrics, Shengjing Hospital of China Medical University, Shenyang, Liaoning 110004, P.R. China
}

Received September 27, 2016; Accepted June 15, 2017

DOI: $10.3892 / \mathrm{etm} .2017 .5055$

\begin{abstract}
Numerous studies have demonstrated that altered expression levels of four and a half LIM domains 1 (FHL1) and P21 are necessary for hypoxia-induced pulmonary vascular remodeling in both adult rats and human patients with idiopathic pulmonary arterial hypertension. However, whether FHL1 and P21 are present in the pulmonary artery and whether these proteins affect pulmonary vascular remodeling in hypoxia-induced pulmonary hypertension (HPH) in neonatal rats remain unknown. The present study investigated the effects of altered FHL1 and P21 expression on pulmonary vascular remodeling in neonatal rats with HPH. A total of 32 newborn Sprague-Dawley rats were exposed to hypoxia or room air for 7 or 14 days ( $\mathrm{n}=8 /$ subgroup). Parameters including the percentage of medial wall thickness (WT\%), the percentage of medial wall area (WA\%), right ventricular (RV) mean pressure, RV hypertrophy index (RVHI) and RV systolic pressure (RVSP) were measured to evaluate the development of HPH. Additionally, the expressions of FHL1 and P21 in the pulmonary artery smooth muscle cells (PASMCs) were measured by reverse transcription-quantitative polymerase chain reaction, western blot analysis and immunohistochemical staining. WA\%, WT\%, RV mean pressure, RVHI and RVSP were significantly increased in the HPH model group
\end{abstract}

Correspondence to: Professor Xindong Xue, Department of Pediatrics, Shengjing Hospital of China Medical University, 36 Sanhao Street, Shenyang, Liaoning 110004, P.R. China E-mail: xuexd@sj-hospital.org

Abbreviations: $\mathrm{PH}$, pulmonary hypertension; PASMCs, pulmonary artery smooth muscle cells; FHL1, four and a half LIM domains 1; $\mathrm{HPH}$, hypoxia-induced pulmonary hypertension; RV, right ventricle; LV, left ventricle; RVHI, right ventricular hypertrophy index; RVSP, right ventricular systolic pressure; WT, medial wall thickness; WA, medial wall area; S, septum; PCNA, proliferating cell nuclear antigen; BPD, bronchoplumonary dysplasia; CDKI, cyclin-dependent kinase inhibitor; HIF, hypoxia-inducible factor; VEGF, vascular endothelial growth factor; TBST, Tris-buffered saline with Tween-20; SD, Sprague-Dawley

Key words: hypoxia-induced pulmonary hypertension, pulmonary vascular remodeling, FHL1, P21, neonatal rats when compared with the control group $(\mathrm{P}<0.01)$. The protein expression levels of FHL1 were significantly increased in the HPH group $(\mathrm{P}<0.05)$, while the mRNA and protein expression levels of $\mathrm{P} 21$ were significantly reduced $(\mathrm{P}<0.05)$. Pearson correlation analysis indicated that the protein expressions of FHL1 and P21 were correlated with WA\% and WT\% (all $\mathrm{P}<0.001$ ), and that the protein expression of $\mathrm{P} 21$ was negatively correlated with that of FHL1 $(\mathrm{P}<0.01)$. The results indicated that the expressions of FHL1 and P21 were altered in the PASMCs of newborn rats with HPH. Furthermore, FHL1 and P21 may serve important roles in pulmonary vascular remodeling.

\section{Introduction}

Pulmonary hypertension (PH) in neonates is a common diagnosis, and is often accompanied by an increase in pulmonary vascular resistance. $\mathrm{PH}$ is considered to be the most frequent complication of chronic neonatal lung disease, particularly in bronchopulmonary dysplasia (BPD) $(1,2)$. Hypoxia is a primary cause of neonatal $\mathrm{PH}$, and hypoxia may induce $\mathrm{PH}$ in adult rats $(3,4)$. In the present study, hypoxic conditions were used to induce $\mathrm{PH}$ in newborn rats. Hypoxia-induced $\mathrm{PH}$ $(\mathrm{HPH})$ in neonates is characterized by an increase in pulmonary vascular resistance and irreversible pulmonary vascular remodeling involving the proliferation of pulmonary artery smooth muscle cells (PASMCs) (5). The pulmonary vascular changes, which mimic those seen in infants with severe BPD, are an important cause of high morbidity and mortality (2). However, the mechanisms that contribute to PASMC proliferation and pulmonary vascular remodeling are not well understood.

Four and a half LIM domains 1 (FHL1) belongs to the Lin-1, Isl-1 and Mec-3 (LIM)-only protein family, and serves vital roles in gene transcription and cell proliferation, differentiation and apoptosis (6). Previous studies have suggested that FHL1 is associated with the development of abnormalities in skeletal muscle and cardiac muscle, including reduced body myopathy (7) and hypertrophic cardiomyopathy (8). Kwapiszewska et al (9) documented that FHL1 was an important factor in pulmonary vascular remodeling in patients with idiopathic pulmonary arterial hypertension, as well as in adult rat models of PH, and that altered expression of FHL1 affected the proliferation and migration of human PASMCs. However, to the best of our knowledge, no study has investigated whether 
FHL1 is related to PASMC proliferation and pulmonary vascular remodeling in newborn rats with $\mathrm{HPH}$.

Cellular proliferation is regulated by a series of cell cycle modulators, including cyclin-dependent kinase inhibitors (CDKIs). P21 ${ }^{\text {Cipl }}$ is one of a few major CDKIs (10). Brock et al (11) reported that, in an HPH mouse model, microRNA-130a controlled the proliferation of pulmonary smooth muscle cells by directly downregulating P21. Furthermore, other studies have demonstrated that rosiglitazone inhibited rat PASMC proliferation by altering the expression levels of heme oxygenase-1 and P21, and that rosiglitazone ameliorated rat pulmonary hypertension by inducing heme oxygenase-1 and P21 expression $(12,13)$. However, whether P21 serves a role in HPH in newborn rats is unknown. Thus, the aim of the current study was to investigate the expressions of FHL1 and P21 in the pulmonary vessels of newborn rats with $\mathrm{HPH}$, as well as the effects of these proteins on pulmonary vascular remodeling.

\section{Materials and methods}

Animal models. All animal experimental procedures and protocols were approved by the Ethics Committee of China Medical University (Shenyang, China). Animals were provided by the Animal Department Experiment Center, Shengjing Hospital of China Medical University (Shenyang, China). A total of 32 newborn Sprague-Dawley (SD) rats (male:female, 1:1; weight, 5-7 g) were randomly assigned to an HPH model group or a control group $(n=16)$. All rats were housed with maternal rats ( $n=4$; weight, 280-320 g; age, 10-12 weeks). Newborn rats in the HPH model group were housed in a hypoxic atmosphere $\left(\mathrm{FiO}_{2}, 0.10\right.$; temperature, $25-27^{\circ} \mathrm{C}$; humidity, $50-70 \%$; $12 \mathrm{~h}$ light/dark cycle), and control rats were housed in a normal air atmosphere $\left(\mathrm{FiO}_{2}, 0.21\right.$; temperature, $25-27^{\circ} \mathrm{C}$; humidity, 50-70\%; $12 \mathrm{~h}$ light/dark cycle) as previously described $(14,15)$. Maternal rats had free access to food and water and were switched between the groups every $24 \mathrm{~h}$ to avoid decreases in feeding ability caused by hypoxia.

Hemodynamic studies and measurement of right ventricular $(R V)$ hypertrophy index. On day 14 of exposure to hypoxic conditions or room air, pups were anesthetized with an intraperitoneal injection of sodium pentobarbital $(50 \mathrm{mg} / \mathrm{kg})$. Tracheal intubation was performed following tracheotomy, and the animals were connected to an animal ventilator (ALC-V8D; Shanghai Alcott Biotech Co., Shanghai, China) to provide breathing support with the following respiratory parameters: Respiratory rate, 100 breaths/min; tidal volume, $0.2 \mathrm{ml}$. Blunt dissection of the left third and fourth intercostal spaces was performed until the heart was fully exposed. The tip of an intravenous trocar was punched into the right ventricle and the opposite end of the trocar was connected to a pressure sensor (BL-420F; Biological Signal Acquisition and Analysis System, Chengdu Taimeng Software Co., Ltd., Chengdu, China) to determine the RV mean pressure and RV systolic pressure (RVSP). The RV free wall was subsequently removed from the left ventricle (LV) and septum (S), and the weights of the RV and the LV plus $\mathrm{S}$ were measured. The RV hypertrophy index (RVHI) was calculated to evaluate the HPH model, and was determined as the ratio of the weight of the
$\mathrm{RV}$ to that of the $\mathrm{LV}$ plus $\mathrm{S}: \mathrm{RV} / \mathrm{LV}+\mathrm{S}$. In addition, RVHI was calculated on day 7 and day 14 of exposure to hypoxia or room air in 8 pups from each subgroup.

Sample preparation and determination of pulmonary vascular remodeling. On days 7 and 14 of exposure to hypoxia or room air, 8 pups from each subgroup were anesthetized with an intraperitoneal injection of sodium pentobarbital (50 mg/kg), and whole lungs were collected. The inferior lobes of the right lungs were fixed in $4 \%$ paraformaldehyde at $4^{\circ} \mathrm{C}$ for $48 \mathrm{~h}$ for hematoxylin and eosin (H\&E) or immunohistochemical staining, while the remaining lungs were stored at $-80^{\circ} \mathrm{C}$ for polymerase chain reaction (PCR) and western blot analyses. The inferior lobes of the right lungs were embedded with paraffin and $4-5-\mu \mathrm{m}$-thick sections were deparaffinized in graded alcohol solutions and xylene, and stained with hematoxylin (at room temperature for $3 \mathrm{~min}$ ) and eosin (at room temperature for $1 \mathrm{~min}$ ) using a staining kit (cat. no. G1120; Beijing Solarbio Science and Technology Co., Ltd., Beijing, China). A total of 3 paraffin-embedded sections were randomly selected from 8 rats/subgroup, and 5 pulmonary arterioles with a diameter of 50 to $100 \mu \mathrm{m}$ from each rat were then selected and images were captured using a light microscope. The pulmonary artery medical wall thickness/external diameter ratio (WT\%) and pulmonary artery medical wall cross-sectional area/total vessel area (WA\%) were analyzed in collected images using Image-Pro Plus 6.0 software (Media Cybernetics, Inc., Rockville, MD, USA) to assess medial wall hypertrophy, as previously described (15).

Immunohistochemistry. Briefly, sections of lung (4-5- $\mu$ m thick) were deparaffinized in a graded series of alcohol and xylene. Following the manufacturer's instructions (immunohistochemistry kit; cat. no. kit-9710; Fuzhou Maixin Biotech Co., Ltd., Fuzhou, China), all sections were subsequently blocked with $3 \% \mathrm{H}_{2} \mathrm{O}_{2}\left(37^{\circ} \mathrm{C}\right.$ for $\left.20 \mathrm{~min}\right)$ and goat serum (provided in kit-9710; $40 \mathrm{~min}$ at room temperature). Sections were subsequently incubated overnight at $4^{\circ} \mathrm{C}$ with FHL1 primary antibody (rabbit anti-rat FHL1, 1:200, cat. no. ab133661), P21 primary antibody (rabbit anti-rat p21, 1:400, cat. no. ab18209) or proliferating cell nuclear antigen (PCNA) primary antibody (rabbit anti-rat PCNA, 1:400, cat. no. ab2426) (all from Abcam, Cambridge, UK). The next day, sections were incubated with biotin-labeled goat anti-rabbit $\operatorname{IgG}$ secondary antibody (provided in kit-9710;20 min at $37^{\circ} \mathrm{C}$ ) and then incubated with a horseradish peroxidase marker (provided in kit-9710; $20 \mathrm{~min}$ at $37^{\circ} \mathrm{C}$ ). The paraffin sections were developed with DAB (30 sec at room temperature, cat. no. ZLI-9017 DAB kit; OriGene Technologies, Inc., Rockville, MD, USA) and counterstained with $10 \%$ hematoxylin for $3 \mathrm{~min}$ at room temperature. Sections were subsequently dehydrated in a graded series of alcohol, treated with xylene and mounted using neutral balsam. A light microscope was used for image acquisition, and the deposition of brown particles indicated a positive result.

Total RNA extraction and reverse transcription-quantitative $P C R(R T-q P C R)$. A total of 8 samples $(60 \mathrm{mg})$ from each group were obtained and total RNA was extracted using TRIzol reagent (Thermo Fisher Scientific, Inc., Waltham, 
Table I. Reverse transcription-quantitative polymerase chain reaction primers.

\begin{tabular}{lll}
\hline Genes & \multicolumn{1}{c}{ Forward (5'-3') } & Reverse $\left(5^{\prime}-3^{\prime}\right)$ \\
\hline FHL1 & CGTGCCAGTAGCGATTCTTAT & GCTGCCTGAAGTGCTTTGAC \\
P21 & CATGTCCGATCCTGGTGATG & CGAACAGACGACGGCATACTT \\
GAPDH & AGACAGCCGCATCTTCTTGT & CTTGCCGTGGGTAGAGTCAT
\end{tabular}

FHL1, four and a half LIM domains 1.

A Rvsp

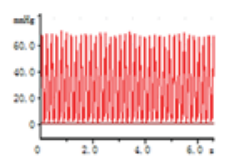

RV mean

pressure

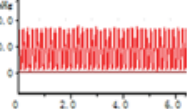

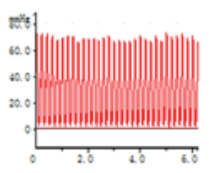

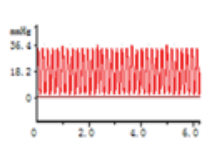

B

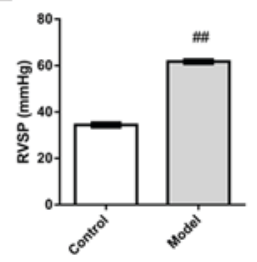

C

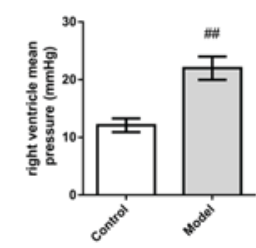

D

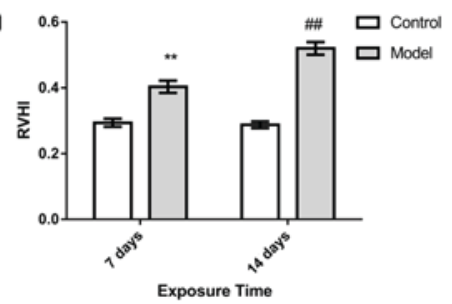

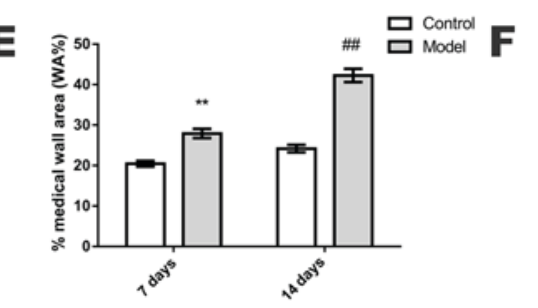

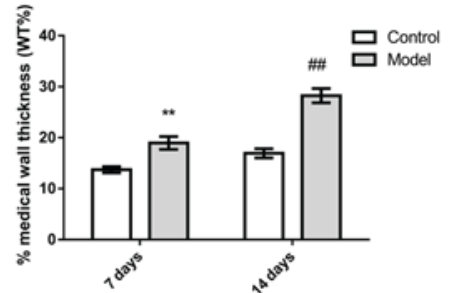

Figure 1. Bar graphs of parameters included in the evaluation model. (A) RV pressure curves; (B) RVSP; (C) RV mean pressure; (D) RVHI, calculated as the weight ratio between the RV and LV plus S: RV/LV+S; (E) medial wall area percentage (WA\%) and (F) medial wall thickness percentage (WT\%) of pulmonary arteries of 50-100 $\mu \mathrm{m}$ in diameter. Bars represent the mean + standard error of the mean, $\mathrm{n}=8 /$ group. ${ }^{* *} \mathrm{P}<0.01$ vs. control at day 7 and ${ }^{\# \#} \mathrm{P}<0.01$ vs. control at day 14. RV, right ventricle/ventricular; LV, left ventricle; RVSP, right ventricular systolic pressure; RVHI, right ventricular hypertrophy index; S, septum.

MA, USA), according to the manufacturer's instructions. RNA purity and concentration were determined according to the OD 260/280 nm ratio. mRNA was reverse transcribed into cDNA using the PrimeScript RT Reagent kit (Takara Biotechnology Co., Ltd., Dalian, China). PCR was performed with a $20 \mu \mathrm{l}$ final volume reaction mixture using a SYBR-Green PCR reagent kit (Takara Biotechnology Co., Ltd.) according to the manufacturer's instructions on the Applied Biosystems 7500 Real-Time PCR system (Applied Biosystems 7500; Thermo Fisher Scientific, Inc.). The cDNA PCR conditions were as follows: 1 cycle of $95^{\circ} \mathrm{C}$ for $30 \mathrm{sec}$, then 40 cycles of $95^{\circ} \mathrm{C}$ for $5 \mathrm{sec}$ and $60^{\circ} \mathrm{C}$ for $34 \mathrm{sec}$. The gene expression levels were calculated with the $2^{-\Delta \Delta \mathrm{Cq}}$ method (16). Glyceraldehyde 3-phosphate dehydrogenase (GAPDH) served as an internal control. The primer sequences are listed in Table I.

Western blotting. The total protein extracted (radioimmunoprecipitation assay lysis buffer, cat. no. P0013B; Beyotime Institute of Biotechnology, Shanghai, China) from lung tissue was quantified using a bicinchoninic acid protein assay kit. A total of $50 \mu \mathrm{g}$ of each protein extract loaded per lane was separated by $12 \%$ SDS-PAGE and transferred to polyvinylidene difluoride membranes (EMD Millipore, Billerica, MA, USA). The membranes were incubated at room temperature for $2 \mathrm{~h}$ in $5 \%$ bovine serum albumin (Beijing Solarbio Science and Technology Co., Ltd., Beijing, China) to block non-specific binding. The membranes were then incubated with primary antibodies against FHL1 (rabbit anti-rat FHL1, 1:400, cat. no. ab133661), P21 (rabbit anti-rat p21, 1:500, cat. no. ab18209) and GAPDH (1:5,000, mouse monoclonal, cat. no. ab9484) (all from Abcam) diluted in Tris-buffered saline with Tween-20 (TBST) at $4^{\circ} \mathrm{C}$ overnight. Following three washes in TBST, the membranes were incubated with a peroxidase-conjugated secondary antibody [goat anti-rabbit immunoglobulin $\mathrm{G}$ (IgG) or goat anti-mouse IgG, 1:2,000; OriGene Technologies, Inc.] for $2 \mathrm{~h}$ at room temperature. The membranes were subsequently imaged using enhanced chemiluminescence reagents (Santa Cruz Biotechnology, Inc., Dallas, TX, USA). The density of the protein bands was analyzed using Image-Pro Plus 6.0 software (Media Cybernetics, Inc.) and normalized to that of GAPDH.

Statistical analysis. Data are presented as the mean + standard error of the mean. Statistical analyses were performed with SPSS 17.0 software (SPSS Inc., Chicago, IL, USA). Student's t-tests were used for inter-group comparisons, and a Pearson's test was used for correlation analysis. $\mathrm{P}<0.05$ was considered to indicate statistical significance.

\section{Results}

$R V$ mean pressure, $R V S P$ and $R V H I$. On day $14, \mathrm{RV}$ mean pressure and RVSP were significantly elevated in the HPH group when compared with the control group $(\mathrm{P}<0.01$; Fig. 1A-C). 


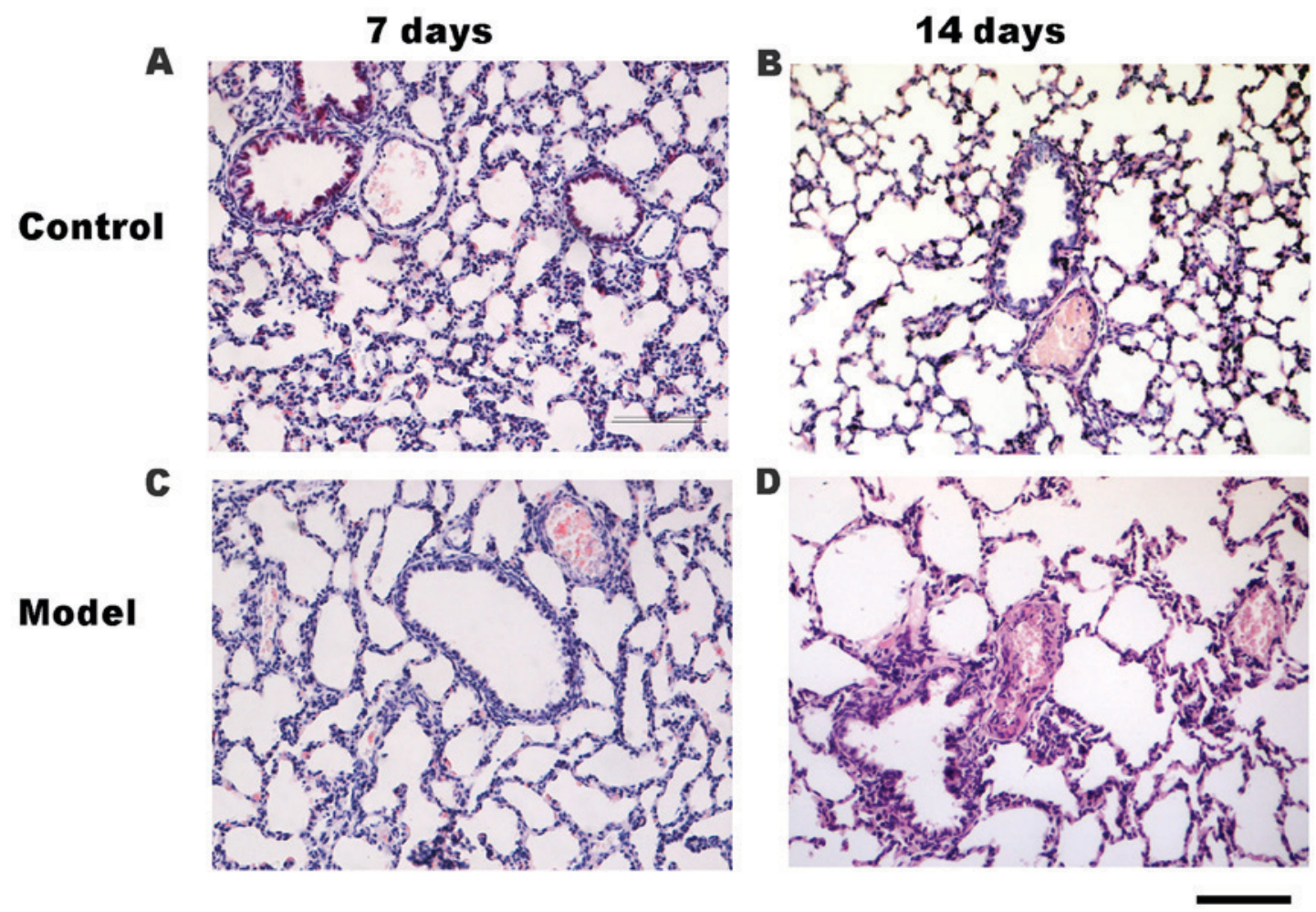

Figure 2. Changes in lung morphology. Lung sections were stained with hematoxylin and eosin, and images were captured under a light microscope (magnification, $\mathrm{x} 20$ ). Representative histological lung sections of the (A and B) control group and (C and D) HPH model group are shown. Lung sections of the HPH group on days 7 and 14 exhibited hypertrophy of the arterial medial wall. Lung histology of the HPH group on day 14 was characterized by increased alveolar space, a thickened alveolar wall, and decreased secondary septa. Scale bar, $100 \mu \mathrm{m}$. HPH, hypoxia-induced pulmonary hypertension.

On days 7 and 14, RVHI was also significantly increased in the HPH group when compared with the control group $(\mathrm{P}<0.01$; Fig. 1D).

$H \& E$ staining and pulmonary vascular remodeling (WA\% and $W T \%$ ). Upon comparison of the small pulmonary arteries (50-100 $\mu \mathrm{m}$ in diameter), the WA\% and WT\% of the HPH group were significantly greater than those in the control group on days 7 and $14(\mathrm{P}<0.01$; Fig. $1 \mathrm{E}$ and $\mathrm{F})$, as determined by H\&E staining (Fig. 2). Accordingly, compared with the control group (Fig. 2A and B), the small pulmonary artery wall was thickened and the lumen was narrowed in the HPH group on days 7 (Fig. 2C) and 14 (Fig. 2D). Additionally, compared with the control group on day 14 (Fig. 2B), the lung histology of rats in the HPH group (Fig. 2D) was characterized by increased alveolar space, a thickened alveolar wall and decreased secondary septa.

Pulmonary artery smooth muscle cell (PASMC) proliferation. The expression of PCNA protein may be used as an indicator of cell proliferation (17), and thus was used to assess the proliferation of PASMCs by immunohistochemical staining. The expression of PCNA protein was identified in the nucleus of PASMCs in both the HPH and control groups (Fig. 3A-D). PCNA expression in the pulmonary arterioles of the HPH group on days 7 (Fig. 3C) and 14 (Fig. 3D) were greater than those in the control group at the same times (Fig. 3A and B).

FHL1 and P21 localization in rat lung tissues following hypoxia. The protein localizations of FHL1 and P21 in the lung tissues were determined by immunohistochemical staining. Only slight positive staining for FHL1 was detected in the small pulmonary arteries of the control group on days 7 (Fig. 4A) and 14 (Fig. 4B). Positive staining for FHL1 was detected in the small pulmonary arteries of the HPH group on days 7 (Fig. 4C) and 14 (Fig. 4D) P21 protein was detected in the nuclei and cytoplasm of PASMCs (Fig. 5A-D). Positive staining for P21 was identified in the cytoplasm of PASMCs in the HPH group on days 7 (Fig. 5C) and 14 (Fig. 5D). However, P21 expression in the pulmonary arterioles of the HPH group on days 7 and 14 (Fig. 5C and D) were markedly lower than those in the control group at the same times (Fig. 5A and B).

Protein expression levels of FHL1 and P21. The protein expression levels of FHL1 and P21 in the lung tissues were determined by western blotting. Compared with the control group, the protein expression of FHL1 was significantly increased in the HPH model group on days $7 \quad(\mathrm{P}<0.01)$ and $14(\mathrm{P}<0.05$; Fig. 6A and $\mathrm{B})$. By contrast, compared with the control group, the protein expression of $\mathrm{P} 21$ was significantly reduced in the HPH group on days $7(\mathrm{P}<0.01)$ and 14 $(\mathrm{P}<0.01$; Fig. 6A and $\mathrm{C})$.

mRNA expression levels of FHL1 and P21. The mRNA expression levels of FHL1 in the lungs did not differ significantly between the control and HPH groups on days 7 and 14 (Fig. 7A). By contrast, compared with the control group, the HPH group exhibited significantly lower levels of P21 mRNA on days 7 and $14(\mathrm{P}<0.05$; Fig. 7B). 

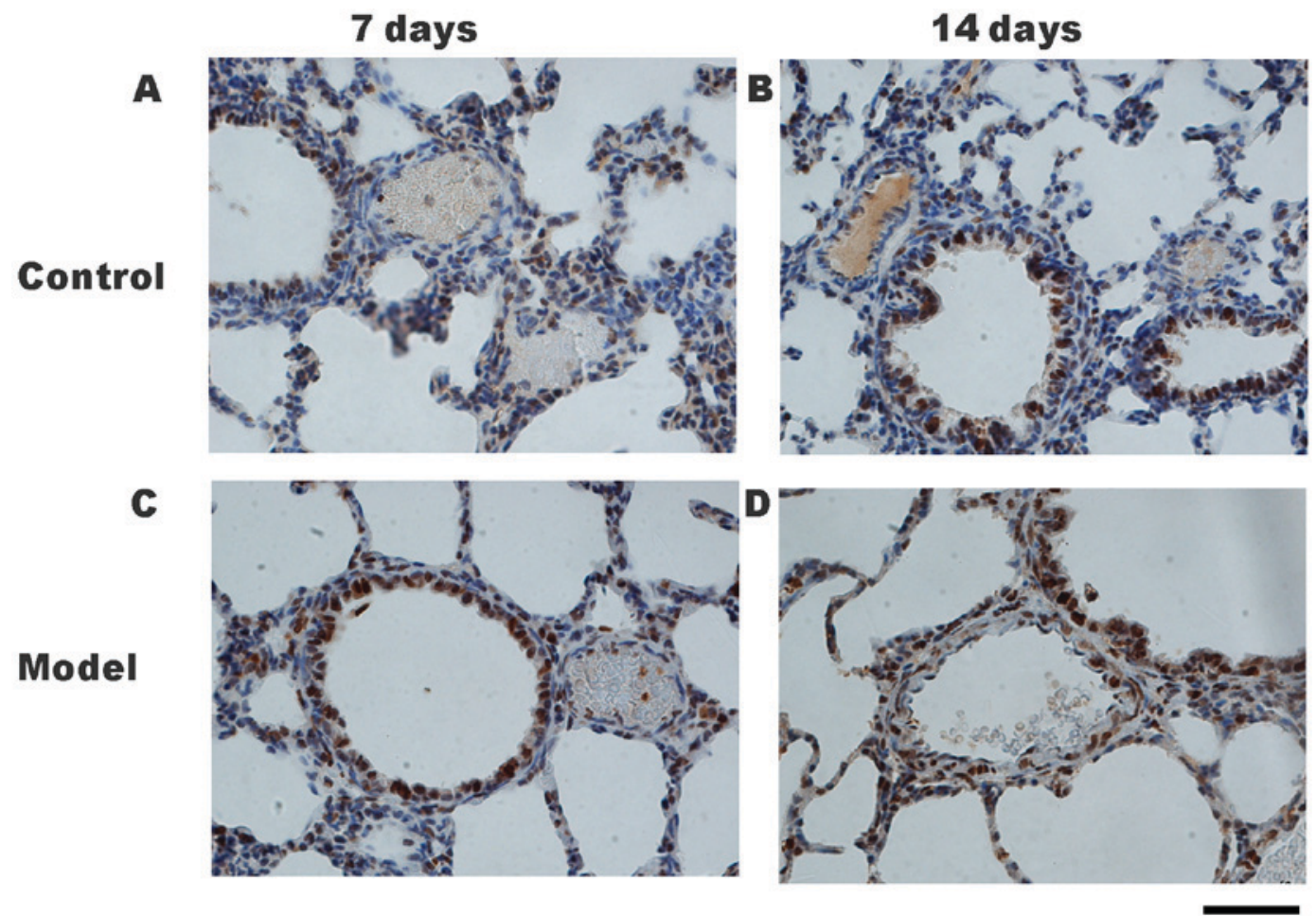

Figure 3. Expression and localization of PCNA in neonatal rats exposed to hypoxia. (A-D) PCNA expression was identified in the nuclei of pulmonary vascular endothelial cells and smooth muscle cells. The relative immunostaining densities for PCNA in the pulmonary arterioles of the (C and D) HPH group were elevated when compared with the (A and B) control group (magnification, $\mathrm{x} 40$ ). Scale bar, $50 \mu \mathrm{m}$. PCNA, proliferating cell nuclear antigen; $\mathrm{HPH}$, hypoxia-induced pulmonary hypertension.

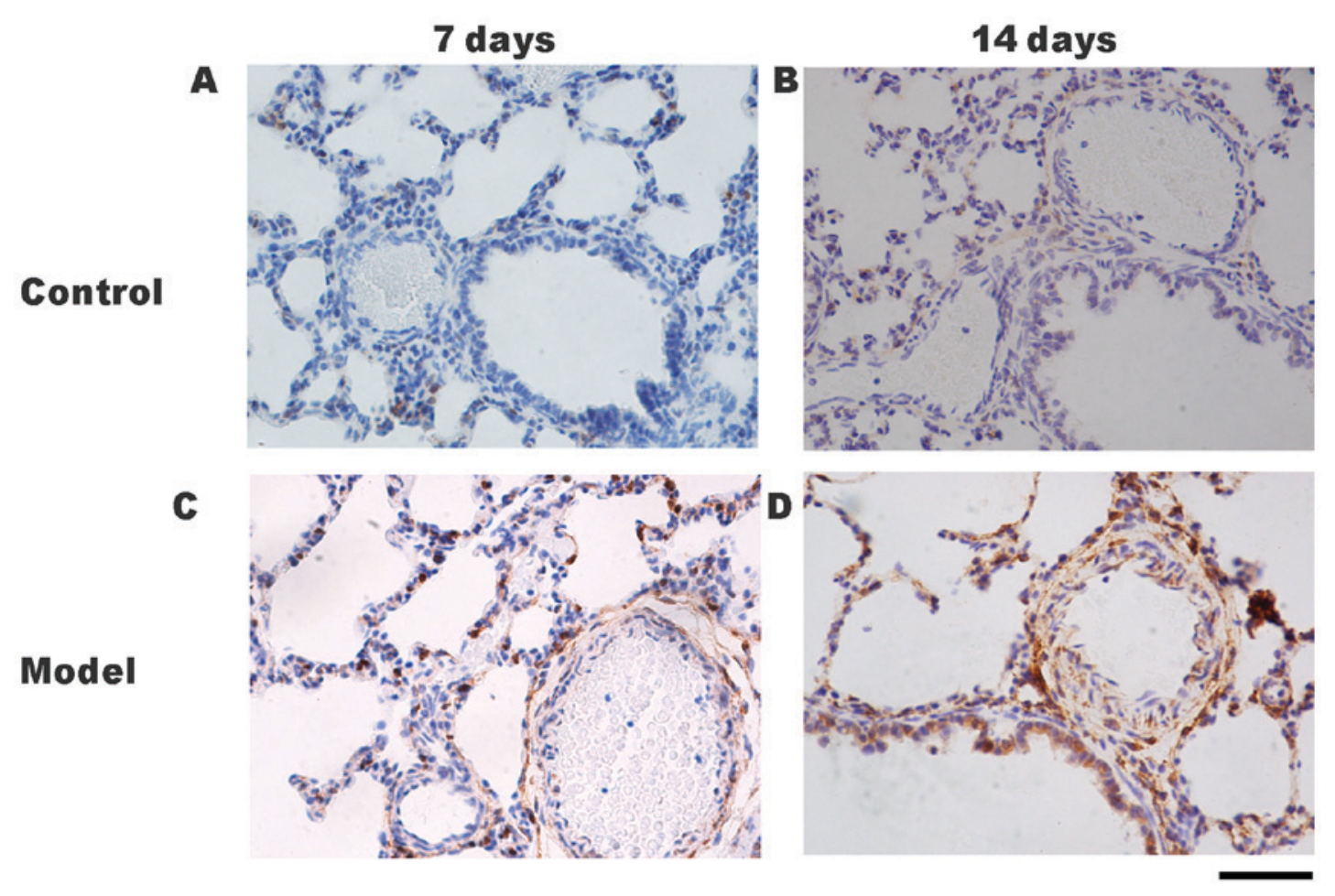

Figure 4. Expression and localization of FHL1 in neonatal rats exposed to hypoxia. (A-D) FHL1 expression was identified in the cytoplasm of the pulmonary artery media and adventitia. (A) In the control group on day 7, FHL1 was marginally expressed. (C) FLH1 was markedly upregulated in the HPH group on day 7. On day 14, the relative immunostaining densities of FHL1 in the pulmonary arterioles of the (D) HPH group were elevated compared with the (B) control group (magnification, x40). Scale bar, $50 \mu \mathrm{m}$. FHL1, four and a half LIM domains 1; HPH, hypoxia-induced pulmonary hypertension.

Associations between FHL1, P21 and HPH. Pearson correlation analysis indicated that the protein expression of FHL1 was negatively associated with the protein expression of P21 ( $r=-0.504, \mathrm{P}<0.01)$. Furthermore, WA\% and WT\% were 

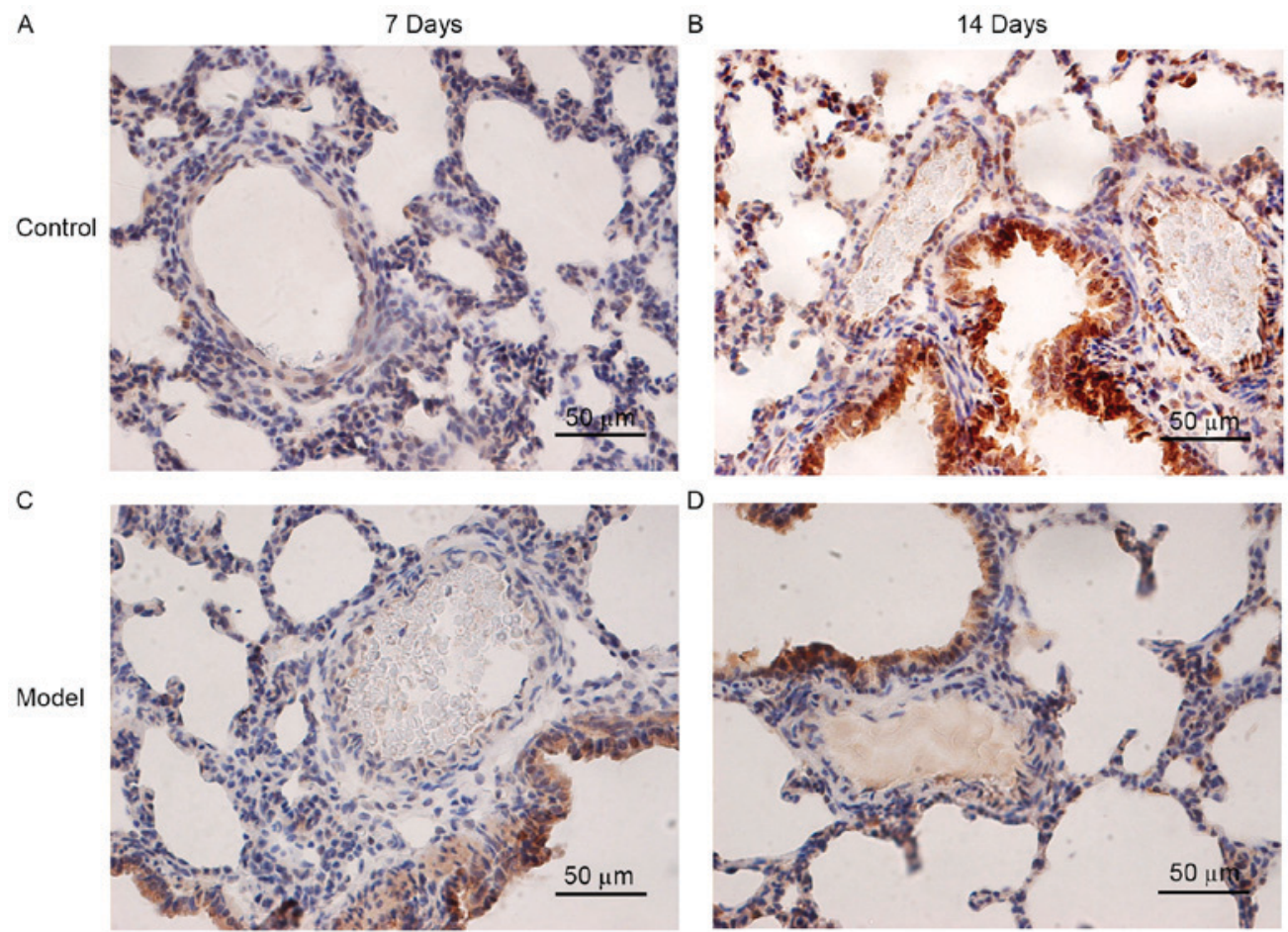

Figure 5. Expression and localization of P21 in neonatal rats exposed to hypoxia. (A-D) P21 expression was identified in the nuclei and cytoplasm of pulmonary vascular endothelial cells and smooth muscle cells. The relative immunostaining densities of P21 in the pulmonary arterioles of the (C and D) HPH group were reduced when compared with the (A and B) control group (magnification, x40). Scale bar, $50 \mu \mathrm{m}$. HPH, hypoxia-induced pulmonary hypertension.

A

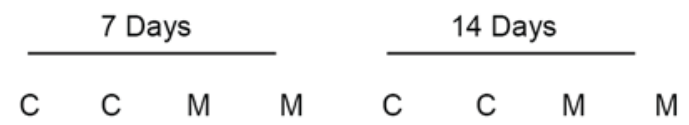

FHL1

$32 \mathrm{kDa}$

P21

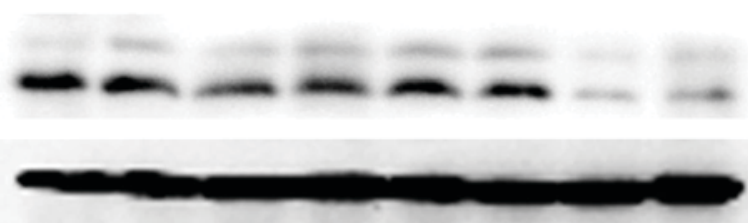

$18 \mathrm{kDa}$

GAPDH

$36 \mathrm{kDa}$
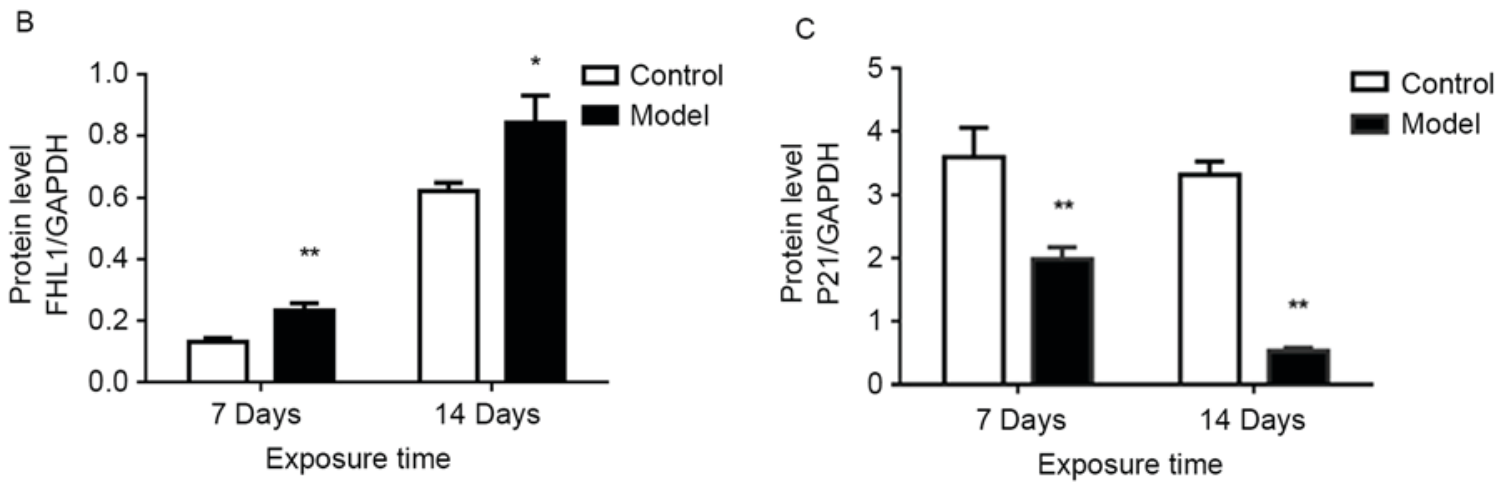

Figure 6. Protein expression levels of FHL1 and P21. (A) The protein expression levels of FHL1 and P21 were measured by western blotting. The intensities of the (B) FHL1 and (C) P21 protein bands were normalized to that of GAPDH. Data are presented as mean + standard error of the mean, $n=8 /$ group. ${ }^{*} \mathrm{P}<0.05$ and ${ }^{* *} \mathrm{P}<0.01$ vs. control. FHL1, four and a half LIM domains 1 .

positively associated with the expression of FHL1 ( $\mathrm{r}=0.627$, $\mathrm{P}<0.001$ and $\mathrm{r}=0.589, \mathrm{P}<0.001$; respectively). Additionally,
WA\% and WT\% were negatively associated with the expression of $\mathrm{P} 21$ ( $\mathrm{r}=-0.805, \mathrm{P}<0.001$ and $\mathrm{r}=-0.749, \mathrm{P}<0.001$; respectively). 
A

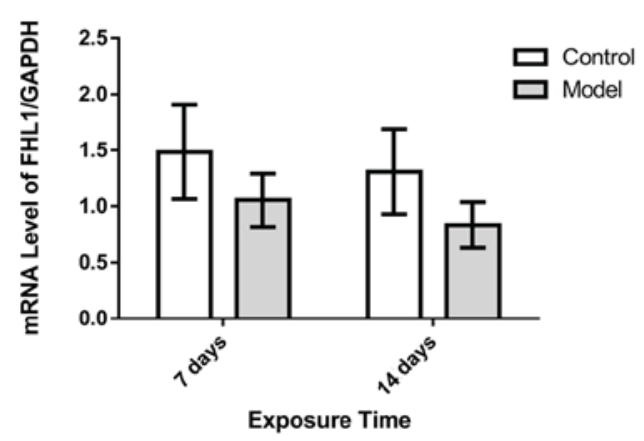

B

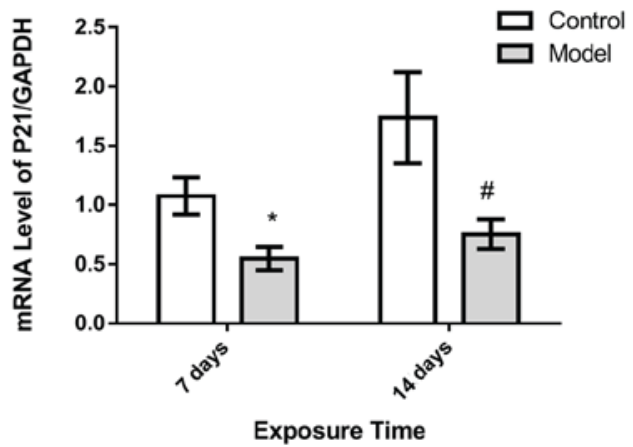

Figure 7. Relative mRNA expression levels of FHL1 and P21. The mRNA expression levels of (A) FHL1 and (B) P21 relative to GAPDH were measured by polymerase chain reaction analysis. Data are presented as mean \pm standard error of the mean, $\mathrm{n}=8 /$ group. ${ }^{*} \mathrm{P}<0.05$ vs. control at day 7 and ${ }^{\#} \mathrm{P}<0.01 \mathrm{vs}$. control at day 14. FHL1, four and a half LIM domains 1.
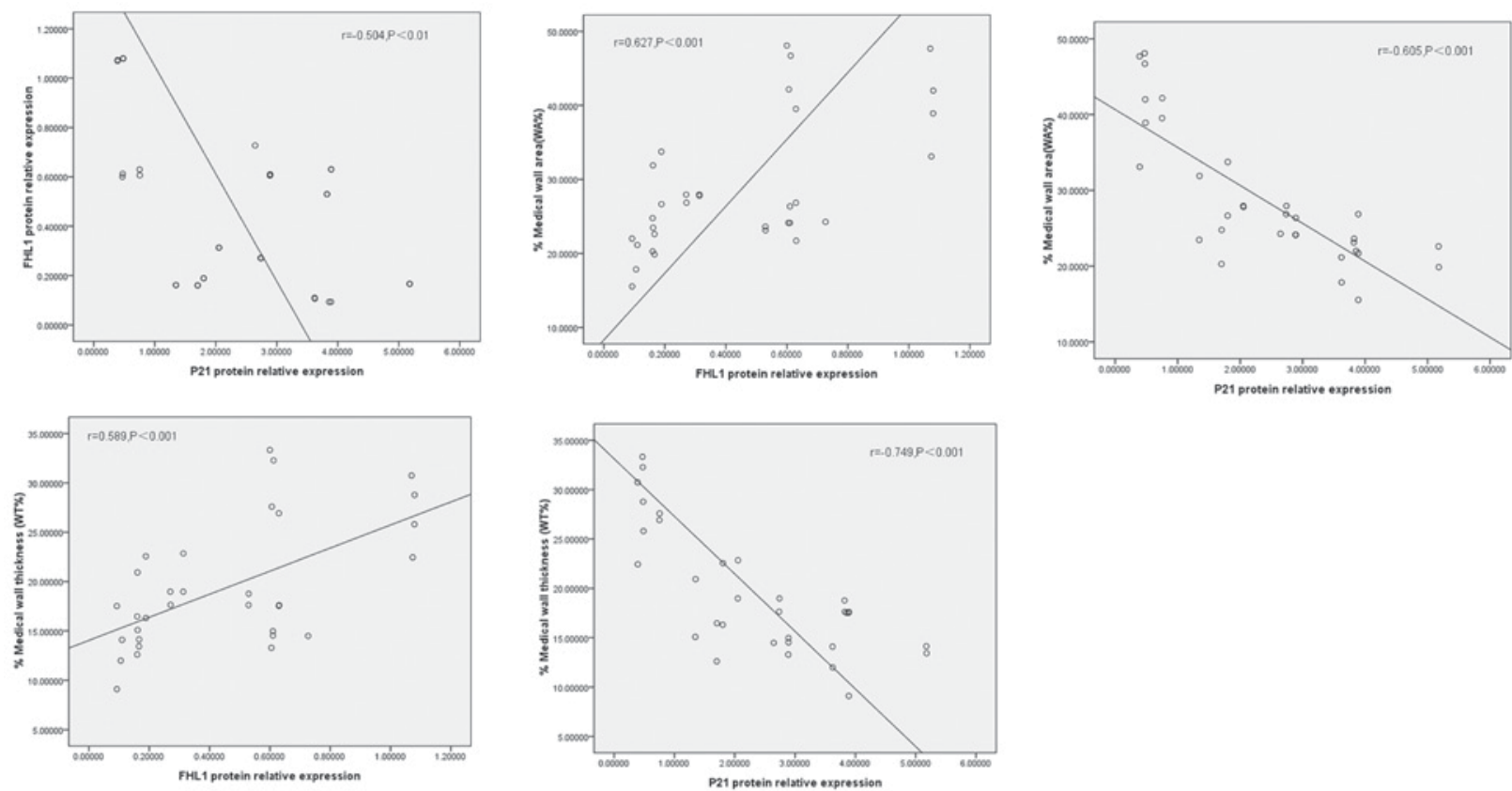

Figure 8. Pearson correlation analysis. The protein expression of $\mathrm{P} 21$ was negatively correlated with that of FHL1 ( $\mathrm{r}=-0.504$, $\mathrm{P}=0.003)$. The protein expressions of FHL1 and P21 were correlated with medial wall area percentage (WA\%; $r=0.627, \mathrm{P}<0.001$ and $\mathrm{r}=-0.805$, $\mathrm{P}<0.001$, respectively) and medial wall thickness percentage (WT\%; $\mathrm{r}=0.589, \mathrm{P}<0.001$ and $\mathrm{r}=-0.749, \mathrm{P}<0.001$, respectively). $\mathrm{n}=8$ /group. FHL1, four and a half LIM domains 1 .

\section{Discussion}

$\mathrm{PH}$ in neonates is a critical disease with a relatively high mortality rate of $10-20 \%$ (18). PH may be triggered by hypoxic lung diseases, including apnea; however, hypoxia is among the main causes of PH $(1,5,19)$. In clinical scenarios, HPH is common; however, if sustained hypoxia is not controlled, hypoxia-induced pulmonary vascular contraction may lead to irreversible pulmonary vascular remodeling, which is associated with a poor prognosis (5).

$\mathrm{HPH}$ is characterized by pulmonary vasoconstriction and irreversible pulmonary vascular remodeling, which involves the proliferation of vascular endothelial cells and PASMCs, and deposition of collagen in the adventitia (20-22). In the present study, 2-day-old SD rats were exposed to hypoxia to induce a model of HPH, and parameters including RV mean pressure, RVHI, WT and WA\% were measured to evaluate the development of HPH. The results demonstrated that RV mean pressure, RVHI, WT and WA\% were significantly increased after 14 days of hypoxia, which was consistent with previous studies $(5,15,23)$. Furthermore, after 14 days of exposure to hypoxia, the lung histology was characterized by increased alveolar space, a thickened alveolar wall and decreased secondary septa. These observations were consistent with a study by Deruelle et al (15). Additionally, immunohistochemical staining indicated that the protein expression of PCNA was significantly higher in the HPH model group than in the control group, which suggested that hypoxia stimulated the proliferation of PASMCs in vitro.

Many factors contribute to the proliferation of PASMCs. For instance, high expression of hypoxia-inducible factor (HIF)-1 $\alpha$ has been related to PASMC proliferation in HPH adult rat models and newborn lamb models of persistent PH (24-26). The present study demonstrated that, in an HPH neonatal rat model, FHL-1 expression was increased in the small pulmonary arteries, which was concurrent with increased PASMC 
proliferation. Kwapiszewska et al (9) documented that FHL1 was markedly upregulated in the lungs of patients with idiopathic pulmonary arterial hypertension, as well as in adult mouse and rat models of HPH. They also observed that knockdown of FHL1 decreased the migration and proliferation of human PASMCs, while FHL1 overexpression had the opposite effects. Furthermore, they concluded that the expression of FHL1 was regulated by HIF- $1 \alpha$ and $-2 \alpha$ (9). Therefore, in the present HPH neonatal rat model, the effect of FHL1 on the proliferation of PASMCs may have been regulated by HIF-1 $\alpha$.

Nadeau et al (27) observed that vascular endothelial growth factor (VEGF) was upregulated in a neonatal pig model of HPH. In human HepG2 hepatoma cells, FHL1 decreased the expression and activity of VEGF by blocking the dimerization of HIF- $1 \alpha$ and HIF-1 $\beta$ (28). Thus, FHL1 may have exerted similar effect in the current HPH neonatal rat model. Additionally, the present data was consistent with previous findings that high expression of FHL1 was correlated with PASMC proliferation induced by cigarette smoke extract (29).

Excessive PASMC proliferation contributes to pulmonary vascular remodeling in HPH $(30,31)$. The cyclin-dependent inhibitor P21 serves an important role in the proliferation of PASMCs, and has been identified as a critical factor in cell cycle progression following exposure to hypoxia (11). However, previous results contradict the potential role of P21 in PH. Yu et al (32) observed that, in an HPH adult mouse model, P27 expression was significantly suppressed, while the expressions of other CDKIs, including P21, were not affected. To clarify the role of FHL1 in cell proliferation, the expression of P21 in the lungs was investigated. Compared with the control group, P21 expression was significantly decreased in the HPH model group. Similarly, a previous study documented that a loss of P21 expression contributed to PASMC proliferation in fetal lambs acclimatized to long-term high-altitude hypoxia (33).

In the present study, the protein expression of FHL1 was significantly increased on days 7 and 14 of hypoxia, while P21 protein expression significantly decreased. Pearson correlation analysis indicated that the protein expressions of FHL1 and P21 were correlated with WA\% and WT\%. Furthermore, the protein expression of $\mathrm{P} 21$ was negatively correlated with that of FHL1. These results suggest that hypoxia increased the expression of FHL1 expression, which subsequently downregulated P21 in the HPH neonatal rat model. In turn, downregulation of P21 may have triggered PASMC proliferation, resulting in pulmonary vascular remodeling.

In conclusion, the present results indicated that the expression profiles of FHL1 and P21 were altered in the PASMCs of neonatal rats with HPH. Additionally, the protein expressions of FHL1 and P21 were correlated with WA\% and WT\%, indicating that FHL1 and P21 may serve important roles in pulmonary vascular remodeling. However, further study is required to verify the roles of FHL1 and P21 in the proliferation of PASMCs in neonatal rats with HPH.

\section{Acknowledgements}

The present study was supported by the Natural Science Foundation of China (grant nos. 81471489 and 81571479).

\section{References}

1. Jain A and McNamara PJ: Persistent pulmonary hypertension of the newborn: Advances in diagnosis and treatment. Semin Fetal Neonatal Med 20: 262-271, 2015.

2. Young KC, Torres E, Hehre D, Wu S, Suguihara C and Hare JM: Antagonism of stem cell factor/c-kit signaling attenuates neonatal chronic hypoxia-induced pulmonary vascular remodeling. Pediatr Res 79: 637-646, 2016.

3. Shao J, Wang P, Liu A, Du X, Bai J and Chen M: Punicalagin prevents hypoxic pulmonary hypertension via anti-oxidant effects in rats. Am J Chin Med 44: 785-801, 2016.

4. Mohsenin V:The emerging role of microRNAsin hypoxia-induced pulmonary hypertension. Sleep Breath 20: 1059-1067, 2016.

5. Wang L, Zhou Y, Li M and Zhu Y: Expression of hypoxia-inducible factor- $1 \alpha$, endothelin-1 and adrenomedullin in newborn rats with hypoxia-induced pulmonary hypertension. Exp Ther Med 8: 335-339, 2014.

6. Zhou Z, Lu J, Dou J, Lv Z, Qin X and Lin J: FHL1 and Smad4 synergistically inhibit vascular endothelial growth factor expression. Mol Med Rep 7: 649-653, 2013.

7. Malfatti E, Olive M, Taratuto AL, Richard P, Brochier G, Bitoun M, Gueneau L, Laforêt P, Stojkovic T, Maisonobe T, et al: Skeletal muscle biopsy analysis in reducing body myopathy and other FHL1-related disorders. J Neuropathol Exp Neurol 72: 833-845, 2013.

8. Friedrich FW, Wilding BR, Reischmann S, Crocini C, Lang P, Charron P, Müller OJ, McGrath MJ, Vollert I, Hansen A, et al: Evidence for FHL1 as a novel disease gene for isolated hypertrophic cardiomyopathy. Hum Mol Genet 21: 3237-3254, 2012.

9. Kwapiszewska G, Wygrecka M, Marsh LM, Schmitt S, Trösser R, Wilhelm J, Helmus K, Eul B, Zakrzewicz A, Ghofrani HA, et al: Fhl-1, a new key protein in pulmonary hypertension. Circulation 118: 1183-1194, 2008.

10. Vermeulen K, Van Bockstaele DR and Berneman ZN: The cell cycle: A review of regulation, deregulation and therapeutic targets in cancer. Cell Prolif 36: 131-149, 2003.

11. Brock M, Haider TJ, Vogel J, Gassmann M, Speich R, Trenkmann M, Ulrich S, Kohler $M$ and Huber LC: The hypoxia-induced microRNA-130a controls pulmonary smooth muscle cell proliferation by directly targeting CDKN1A. Int J Biochem Cell Biol 61: 129-137, 2015.

12. Li M, Li Z, Sun X, Yang L, Fang P, Liu Y, Li W, Xu J, Lu J, Xie M and Zhang D: Heme oxygenase-1/p21WAF1 mediates peroxisome proliferator-activated receptor-gamma signaling inhibition of proliferation of rat pulmonary artery smooth muscle cells. FEBS J 277: 1543-1550, 2010.

13. Zhang D, Wang G, Han D, Zhang Y, Xu J, Lu J, Li S, Xie X, Liu L, Dong L and Li M: Activation of PPAR- $\gamma$ ameliorates pulmonary arterial hypertension via inducing heme oxygenase-1 and p21 (WAF1): An in vivo study in rats. Life Sci 98: 39-43, 2014.

14. Xu XF, Gu WZ, Wu XL, Li RY and Du LZ: Fetal pulmonary vascular remodeling in a rat model induced by hypoxia and indomethacin. J Matern Fetal Neonatal Med 24: 172-182, 2011.

15. Deruelle P, Balasubramaniam V, Kunig AM, Seedorf GJ, Markham NE and Abman SH: BAY 41-2272, a direct activator of soluble guanylate cyclase, reduces right ventricular hypertrophy and prevents pulmonary vascular remodeling during chronic hypoxia in neonatal rats. Biol Neonate 90: 135-144, 2006.

16. Livak KJ and Schmittgen TD: Analysis of relative gene expression data using real-time quantitative PCR and the 2(-Delta Delta C(T)) Method. Methods 25: 402-408, 2001.

17. Xiang H, Yuan L, Gao X, Alexander PB, Lopez O, Lau C, Ding Y, Chong M, Sun T, Chen R, et al: UHRF1 is required for basal stem cell proliferation in response to airway injury. Cell Discov 3: 17019, 2017.

18. Perez KM and Laughon M: Sildenafil in term and premature infants: A systematic review. Clin Ther 37: 2598-2607, 2015.

19. Steinhorn RH: Advances in neonatal pulmonary hypertension. Neonatology 109: 334-344, 2016.

20. He Y, Cao X, Liu X, Li X, Xu Y, Liu J and Shi J: Quercetin reverses experimental pulmonary arterial hypertension by modulating the TrkA pathway. Exp Cell Res 339: 122-134, 2015.

21. Li L, Wang X, Wang L, Qu L, Zhu X, Li M, Dang X, Li P, Gao Y, Peng Z, et al: Mammalian target of rapamycin overexpression antagonizes chronic hypoxia-triggered pulmonary arterial hypertension via the autophagic pathway. Int J Mol Med 36: 316-322, 2015. 
22. Umesh A, Paudel O, Cao YN, Myers AC and Sham JS: Alteration of pulmonary artery integrin levels in chronic hypoxia and monocrotaline-induced pulmonary hypertension. J Vasc Res 48: 525-537, 2011

23. Xu YP, Zhu JJ, Cheng F, Jiang KW, Gu WZ, Shen Z, Wu YD, Liang L and Du LZ: Ghrelin ameliorates hypoxia-induced pulmonary hypertension via phospho-GSK3 $\beta / \beta$-catenin signaling in neonatal rats. J Mol Endocrinol 47: 33-43, 2011.

24. Yue J, Guan J, Wang X, Zhang L, Yang Z, Ao Q, Deng Y, Zhu P and Wang G: MicroRNA-206 is involved in hypoxia-induced pulmonary hypertension through targeting of the HIF-1 $\alpha / \mathrm{Fhl}-1$ pathway. Lab Invest 93: 748-759, 2013.

25. Zhang L, Pu Z, Wang J, Zhang Z, Hu D and Wang J: Baicalin inhibits hypoxia-induced pulmonary artery smooth muscle cel proliferation via the AKT/HIF-1 $\alpha / \mathrm{p} 27$-associated pathway. Int J Mol Sci 15: 8153-8168, 2014.

26. Wedgwood S, Lakshminrusimha S, Schumacker PT and Steinhorn RH: Hypoxia inducible factor signaling and experimental persistent pulmonary hypertension of the newborn. Front Pharmacol 6: 47, 2015.

27. Nadeau S, Baribeau J, Janvier A and Perreault T: Changes in expression of vascular endothelial growth factor and its receptors in neonatal hypoxia-induced pulmonary hypertension. Pediatr Res 58: 199-205, 2005.

28. Lin J, Qin X, Zhu Z, Mu J, Zhu L, Wu K, Jiao H, Xu X and Ye Q: FHL family members suppress vascular endothelial growth factor expression through blockade of dimerization of HIF1 $\alpha$ and HIF1ß. IUBMB Life 64: 921-930, 2012.
29. Li Y, Pu G, Chen C and Yang L: Inhibition of FHL1 inhibits cigarette smoke extract-induced proliferation in pulmonary arterial smooth muscle cells. Mol Med Rep 12: 3801-3808, 2015

30. Huetsch JC, Jiang $\mathrm{H}$, Larrain $\mathrm{C}$ and Shimoda LA: The $\mathrm{Na}^{+} / \mathrm{H}^{+}$ exchanger contributes to increased smooth muscle proliferation and migration in a rat model of pulmonary arterial hypertension. Physiol Rep 4: e12729, 2016.

31. Wei C, Li HZ, Wang YH, Peng X, Shao HJ, Li HX, Bai SZ, $\mathrm{Lu} \mathrm{XX}, \mathrm{Wu} \mathrm{LY}$, Wang $\mathrm{R}$ and $\mathrm{Xu} \mathrm{CQ}$ : Exogenous spermine inhibits the proliferation of human pulmonary artery smooth muscle cells caused by chemically-induced hypoxia via the suppression of the ERK1/2- and PI3K/AKT-associated pathways. Int J Mol Med 37: 39-46, 2016.

32. Yu L, Quinn DA, Garg HG and Hales CA: Gene expression of cyclin-dependent kinase inhibitors and effect of heparin on their expression in mice with hypoxia-induced pulmonary hypertension. Biochem Biophys Res Commun 345: 1565-1572, 2006.

33. Yang Q, Lu Z, Ramchandran R, Longo LD and Raj JU: Pulmonary artery smooth muscle cell proliferation and migration in fetal lambs acclimatized to high-altitude long-term hypoxia: Role of histone acetylation. Am J Physiol Lung Cell Mol Physiol 303: L1001-L1010, 2012. 